\title{
Characteristic functions of differential equations with deviating arguments.
}

\author{
Christopher T H Baker ${ }^{\mathrm{a}}$, Neville J Ford ${ }^{\mathrm{b}, *}$ \\ ${ }^{a}$ Formerly Emeritus professor, University of Chester and Emeritus professor, University of Manchester. \\ ${ }^{b}$ University of Chester, Parkgate Road, Chester, CH1 4BJ, UK.
}

\section{Abstract}

The material here is motivated by the discussion of solutions of linear homogeneous and autonomous differential equations with deviating arguments. If $a, b, c$ and $\left\{\check{\tau}_{\ell}\right\}$ are real and $\gamma_{\natural}$ is real-valued and continuous, an example with these parameters is

$$
u^{\prime}(t)=\left\{a u(t)+b u\left(t+\check{\tau}_{1}\right)+c u\left(t+\check{\tau}_{2}\right)\right\}+\int_{\check{\tau}_{3}}^{\check{\tau}_{4}} \gamma_{\mathfrak{G}}(s) u(t+s) d s .
$$

A wide class of equations $(\star)$, or of similar type, can be written in the "canonical" form

$$
u^{\prime}(t)=\int_{\tau_{\min }}^{\tau_{\max }} u(t+s) d \sigma(s) \quad(t \in \mathbb{R}), \text { for a suitable choice of } \tau_{\min }, \tau_{\max }
$$

where $\sigma$ is of bounded variation and the integral is a Riemann-Stieltjes integral. For equations written in the form $(\star \star)$, there is a corresponding characteristic function

$$
\chi(\zeta)):=\zeta-\int_{\tau_{\min }}^{\tau_{\max }} \exp (\zeta s) d \sigma(s) \quad(\zeta \in \mathbb{C}),
$$

whose zeros (if one considers appropriate subsets of equations $(\star \star)$ - the literature provides additional information on the subsets to which we refer) play a rôle in the study of oscillatory or non-oscillatory solutions, or of bounded or unbounded solutions. We show that the related discussion of the zeros of $\chi$ is facilitated by observing and exploiting some simple and fundamental properties of characteristic functions.

Keywords: Differential equations with deviating arguments, equations of mixed-type, characteristic functions, discretizations, oscillatory and non-oscillatory and bounded or unbounded functions.

\section{Introduction and statement on authorship}

This review paper was first motivated by discussions between the authors (CTHB and NJF) when the papers [4] and [5] were in preparation. The discussions focused on retarded and mixedtype functional differential equations and exposed the very complex dynamical behaviours found in these problems and the fact that insights from retarded differential equations provide an inadequate basis for understanding the dynamics of equations of these different types. The discussions also concerned the related papers [3, 11, 12. As a consequence of these discussions, one author CTHB began to prepare a review paper on the subject.

Sadly, CTHB passed away in 2017, leaving an unfinished manuscript, which his co-author NJF has completed, on the invitation of CTHB's family, to enable its publication.

\footnotetext{
* Corresponding author

Email address: njford@chester.ac.uk (Neville J Ford)
} 


\section{Prologue}

\subsection{Case study: an equation studied by E.C. Titchmarsh}

We open with a simple (symmetric) example of the type of equation $\left(^{*}\right)$ to be considered.

Example 2.1. In the paper [13] read to the London Mathematical Society in February 1939, Titchmarsh illustrated how Fourier integrals can be modified to determine, without assuming exponential boundedness of $y$, solutions of the equation $y^{\prime}(t)=\frac{\{y(t+h)-y(t-h)\}}{2 h}$ with $h>0$. One can normalize the problem and chose $h=1$, so we address

$$
u^{\prime}(t)=\frac{1}{2}\{u(t+1)-u(t-1)\} \quad(t \in \mathbb{R})
$$

where $u(t)=y(t / h)$ (so we reformulate the results in [13]). From Titchmarsh [13, equation (5)], we conclude that every complex-valued solution $u$ may be expressed for suitable scalars $A, B, C,\left\{C_{\ell}\right\}$ in the form

$$
u(t)=A+B t+C t^{2}+\sum_{\xi_{\ell} \neq 0} C_{\ell} \exp \left(\zeta_{\ell} t\right)
$$

where the sum runs over non-zero values $\zeta_{\ell}$ satisfying

$$
\chi\left(\zeta_{\ell}\right)=0, \text { with } \chi(\zeta):=\zeta-\sinh (\zeta)
$$

and $\sinh (\zeta) \equiv \frac{1}{2}[\exp (\zeta)-\exp (-\zeta)]$ for $\zeta \in \mathbb{C}$. The real and imaginary parts of 2.2 provide realvalued solutions. The value $\zeta_{0}=0$ is a zero of $\chi$ of multiplicity three since $\chi(0)=\chi^{\prime}(0)=\chi^{\prime \prime}(0)=0$ and $\chi^{\prime \prime \prime}(0) \neq 0$. In 2.2 , the term $A+B t+C t^{2}$ is, in fact, $\left(A+B t+C t^{2}\right) \exp \left(\zeta_{0} t\right)$. The non-zero characteristic values $\left\{\zeta_{\ell}\right\}$ are simple zeros and collectively give rise to the sum $\sum_{\xi_{\ell} \neq 0} C_{\ell} \exp \left(\xi_{\ell} t\right)$. A little analysis reveals that any non-zero characteristic value $\left\{\zeta_{\ell}\right\}$ has a non-zero imaginary part (and its complex conjugate is also a characteristic value). Equation 2.2) is an example of a solution expansion

$$
u(t)=\sum_{\ell=0}^{\infty} P_{\ell}(t) \exp \left(\xi_{\ell} t\right) \text { for } t \geq 0
$$

(cf. (7.13)). The reader will observe that Titchmarsh proves that all complex solutions have the representation (2.2). The discussion of Titchmarsh served to illustrate a technique based on modified Fourier transforms that avoids usual assumptions of transform theory.

\section{Preliminaries}

\subsection{Some basic material}

Let $\mathfrak{F}$ denote a linear space of real-valued functions of a real variable $t \in \mathbb{R}$. Suppose that elements of $\mathfrak{F}$ are continuous on $\mathbb{R}$ (the continuity requirement may be strengthened).

Definition 3.1. We denote by $\mathfrak{A}$ the set of operators $\mathcal{L}$ on $\mathfrak{F}$ that are linear and autonomous:

$$
\begin{gathered}
\mathcal{L}\left\{a_{0} u_{0}+a_{1} u_{1}\right\}(t)=a_{0} \mathcal{L} u_{0}(t)+a_{1} \mathcal{L} u_{1}(t), \quad\left(\forall a_{0}, a_{1}, t \in \mathbb{R}\right) \text { when } u_{0}, u_{1} \in \mathfrak{F}, \\
\mathcal{L} u(t+s)=\mathcal{L} u(t) \quad(\forall s, t \in \mathbb{R}) \quad \text { when } u \in \mathfrak{F} .
\end{gathered}
$$

$\mathcal{L} u(t)$ is synonymou $\underbrace{1}$ with $\mathcal{L}\{u\}(t)$.

Next we recall a definition from basic analysis.

\footnotetext{
${ }^{1}$ In applications, $t$ need not represent time. By an abuse of notation we sometime write "the function $u(t)$ " for " the function $u$ with argument $t "$.
} 
Definition 3.2 (Total variation, bounded variation). For a function $\sigma \in\left(\left[-\tau_{\natural}, \tau^{\natural}\right] \rightarrow \mathbb{R}\right)$, the total variation $\mathrm{V}_{-\tau_{\natural}} \tau^{\natural}(\sigma)$ is $\sup _{\left\{r_{\ell}\right\}} \sum_{0}^{N-1}\left|\sigma\left(r_{i+1}\right)-\sigma\left(r_{i}\right)\right|$ where the supremum is taken over all partitions $\left\{r_{\ell}\right\}$ of $\left[-\tau_{\natural}, \tau^{\natural}\right]$, each with $-\tau_{\natural}=r_{0}<r_{1}<\cdots<r_{N}=\tau^{\natural}$ for some integer $N$. Functions $\sigma$ of bounded variation are those functions with $\mathrm{V}_{-\tau_{\natural}^{\natural}}^{\tau^{\natural}}(\sigma)<\infty$.

We suppose $\mathfrak{N}_{1}$ and $\mathfrak{N}_{2}$ are finite sets of integers, the real values $\left\{\tau_{j}\right\}_{j \in \mathfrak{N}_{1}}$ are enumerated algebraically, $\gamma_{1, j} \in \mathbb{R}$ for $j \in \mathfrak{N}_{1}$, and the real-valued function $\gamma_{2, k}$ is continuous on $\left[\widehat{\tau}_{k}, \widehat{\tau}^{k}\right]$ for $k \in \mathfrak{N}_{2}$.

Definition 3.3. We write $\mathcal{L} \in \mathfrak{A}_{\sqrt{3.2}}$ if

$$
\mathcal{L} u(t)=\sum_{j \in \mathfrak{N}_{1}} \gamma_{1, j} u\left(t-\tau_{j}\right)+\sum_{k \in \mathfrak{N}_{2}} \int_{\widehat{\tau}_{k}}^{\widehat{\tau}^{k}} u(t-s) \gamma_{2, k}(s) d(s) \quad(t \in \mathbb{R}) .
$$

If there exists a function $\sigma$ of bounded variation on an interval $\left[\tau_{\min }, \tau_{\max }\right]$ and

$$
\mathcal{L} u(t)=\int_{\tau_{\min }}^{\tau_{\max }} u(t+s) d \sigma(s) \quad(t \in \mathbb{R})
$$

then we write $d \sigma=d \sigma(\mathcal{L})$ and $\mathcal{L} \in \mathfrak{A}_{3.31}$.

To extend $\mathcal{L}$ to a space of complex-valued functions $w \in\left(\mathbb{R} \rightarrow \mathbb{C}\right.$ ), we write (when $w_{0}, w_{1} \in \mathfrak{F}$ where $\left.w_{0}(t)=\Re w(t), w_{1}(t)=\Im w(t)\right)$,

$$
\mathcal{L} w(t):=\mathcal{L}(\Re\{w(t)\})+\mathrm{i} \mathcal{L}(\Im\{w(t)\}) \quad(\mathrm{i}=\sqrt{-1}) \text { for } t \in \mathbb{R} .
$$

Equations with $\tau_{\min }=-\tau_{\max }$ often occur in the literature ( $c f$. Example 2.1).

Remark 3.1. If we select $s_{*} \in\left[\tau_{\min }, \tau_{\max }\right]$ then, given $d \sigma$ we can rewrite 3.3 in the form

$$
\mathcal{L} u(t)=a_{*} u\left(t+s_{*}\right)+\int_{\tau_{\min }}^{\tau_{\max }} u(t+s) d \sigma_{*}(s) \quad(t \in \mathbb{R}),
$$

where $\sigma_{*}$ is continuous at $s_{*}\left(a_{*} \in \mathbb{R}\right.$ may be zero $)$.

\subsection{Types of equation}

Lemma 3.1. $\mathfrak{A}_{(3.2)}=\mathfrak{A}_{(3.3)}$ in the sense that every equation 3.2 can be expressed in the form (3.3) for some $\sigma$ of bounded variation that is differentiable almost everywhere (having a finite set of jump discontinuities), and vice versa.

We indicate a proof. Any bounded linear autonomous operator $\mathscr{L}$ on $\mathfrak{F}$ generates a corresponding linear functional $\Lambda$ on setting $\Lambda u=\mathscr{L}\{u\}(0)$ and, conversely, every bounded linear functional $\Lambda$ generates a bounded linear autonomous operator $\mathscr{L}$. The Riesz representation theorem for $\Lambda$ provides a corresponding representation for $\mathscr{L}$ and this representation theorem, when applied to $\mathcal{L}$, leads us to formulate the preceding result. Its significance lies in the fact that our theoretical discussion can be focussed, should we wish, on the form (3.3). Given an example of the form (3.2), we can determine a corresponding $\sigma$.

Remark 3.2. With an obvious notation, we can define $\left\{\mathcal{L}_{1, j}\right\}_{j \in \mathfrak{N}_{1}}$ and $\left\{\mathcal{L}_{2, k}\right\}_{k \in \mathfrak{N}_{2}}$ corresponding to 3.2 so that 3.2 reads $\mathcal{L} u(t)=\sum_{j \in \mathfrak{N}_{1}}\left\{\mathcal{L}_{1, j} u(t)\right\}+\sum_{k \in \mathfrak{N}_{2}}\left\{\mathcal{L}_{2, k} u(t)\right\}$ and

$$
\mathcal{L}=\sum_{j \in \mathfrak{N}_{1}} \mathcal{L}_{1, j}+\sum_{k \in \mathfrak{N}_{2}} \mathcal{L}_{2, k}
$$

If we take $\left[\tau_{\min }, \tau_{\max }\right]$ to be the minimal connected region that contains $\left\{\tau_{j}\right\}$ and the intervals $\left[\widehat{\tau}_{k}, \widehat{\tau}^{k}\right]$ and we identify $d \sigma_{1, j} \equiv d \sigma\left(\mathcal{L}_{1, j}\right)$ and $d \sigma_{2, k} \equiv d \sigma\left(\mathcal{L}_{2, j}\right)$ so that

$$
\mathcal{L}_{1, j} u(t)=\int_{\tau_{\min }}^{\tau_{\max }} u(t+s) d \sigma_{1, j}(s) \text { and } \mathcal{L}_{2, k} u(t)=\int_{\tau_{\min }}^{\tau_{\max }} u(t+s) d \sigma_{2, k}(s)
$$

then (3.6) gives $d \sigma(\mathcal{L})=\sum_{j}\left\{d \sigma\left(\mathcal{L}_{1, j}\right)\right\}+\sum_{k}\left\{d \sigma\left(\mathcal{L}_{2, k}\right)\right\}$. 
Denote by $\mathfrak{F}^{\prime}$ the subset of $\mathfrak{F}$ consisting of functions differentiable on $\mathbb{R}$, and write

$$
\mathcal{D} u(t)=u^{\prime}(t) \quad(t \in \mathbb{R}), \text { for } u \in \mathfrak{F}^{\prime} .
$$

This definition extends to complex-valued diferentiable functions; compare (3.4). The restriction of $\mathcal{L}$ to $\mathfrak{F}^{\prime}$ is also denoted $\mathcal{L}$. and we define a linear operator $\mathcal{M}$ on $\mathfrak{F}^{\prime}$ with

$$
\mathcal{M}:=\mathcal{D}-\mathcal{L} .
$$

The "differential equations" of interest, $u^{\prime}(t)=\mathcal{L} u(t)$, can be represented in the form $\mathcal{M}\{u\}=0$, or $\mathcal{M} u(t)=0$ for $t \in \mathbb{R}$. Thus, $(\star \star)$ can be written $\mathcal{M} u(t)=0$ where $\mathcal{M}=\mathcal{D}-\mathcal{L}$ and $\mathcal{L} \in \mathfrak{A}_{(3.3)}$. We suppose $\tau_{\min }<\tau_{\max }$ but do not assume $\tau_{\min } \times \tau_{\max } \leq 0$.

Definition 3.4 (Retarded, advanced, and mixed-type problems). Various subclasses of problem appear in the literature:

1. (Class $O)$ ordinary differential equations, where $s_{*}=0$ and $d \sigma_{*}(s) \equiv 0$ in 3.5 ;

2. (Class D:) delayed or retarded problems, where $\left[\tau_{\min }, \tau_{\max }\right] \cap(0, \infty)$ is empty;

3. (Class A:) advanced problems, where $\left[\tau_{\min }, \tau_{\max }\right] \cap(-\infty, 0)$ is empty;

4. (Class M:) problems of mixed type, where neither (1) nor (2) apply.

Class $D$ corresponds to $d \sigma(t)=0$ for $t>0$, Class $A$ corresponds to $d \sigma(t)=0$ for $t<0$.

Class D has attracted most discussion in the literature. We shall discuss further classes of equation later.

\section{Characteristic functions, values, and solutions}

The characteristic functions that we associate with $(\star)$ and $(\star \star)$ in the abstract are

$$
\begin{gathered}
\zeta-\left\{a+b \exp \left(\check{\tau}_{1} \zeta\right)+c \exp \left(\check{\tau}_{2} \zeta\right)+\int_{\check{\tau}_{3}}^{\check{\tau}_{4}} \gamma_{\natural}(s) \exp (s \zeta) d s\right\} \quad(\zeta \in \mathbb{C}) ; \\
\zeta-\int_{\tau_{\min }}^{\tau_{\max }} \exp (\zeta s) d \sigma(s) .
\end{gathered}
$$

We make the concept of characteristic function precise. $\mathcal{L}$ may be any linear autonomous operator on $\mathfrak{F}$ but suppose $\mathcal{L} \in \mathfrak{A}_{\sqrt{3.2}}$ or $\mathcal{L} \in \mathfrak{A}_{[3.3}$. Define

$$
\chi_{\mathcal{L}}(\zeta)=\exp (-\zeta t) \mathcal{L} \exp (-\zeta t) \text { for arbitrary } t \in \mathbb{R} .
$$

as the characteristic function of $\mathcal{L}$. Similarly, for $(3.9)\left(\mathcal{M}\right.$ defined on $\left.\mathfrak{F}^{\prime}\right)$, define

$$
\chi_{\mathcal{M}}(\zeta)=\exp (-\zeta t) \mathcal{M} \exp (-\zeta t) \quad(t \in \mathbb{R} \text { arbitrary })
$$

the characteristic function of $\mathcal{M}$. Equivalently,

$$
\chi_{\mathcal{M}}(\zeta):=\zeta-\chi_{\mathcal{L}}(\zeta)
$$

Specifically, for $\mathcal{L} \in \mathfrak{A}_{\sqrt{3.3}}, \chi_{\mathcal{M}}(\zeta)$ reduces to $\left[4.2\right.$. The function $\chi_{\mathcal{M}}$ in 4.5 is analytic and its realor complex-valued zeros are called the characteristic values of $\mathcal{M}$. The set of characteristic values $\left\{\zeta_{\ell}\right\}$ of $\mathcal{M}$ is countable, and will be denoted $\Sigma(\mathcal{M})$. We have

$$
\zeta_{\ell}^{ \pm}=\xi_{\ell} \pm \mathrm{i} \eta_{\ell}, \quad \xi_{\ell}=\int_{\tau_{\min }}^{\tau_{\max }} \exp \left(\xi_{\ell} s\right) \cos \left(\eta_{\ell}\right) d \sigma(s), \quad \eta_{\ell}=\int_{\tau_{\min }}^{\tau_{\max }} \exp \left(\xi_{\ell} s\right) \sin \left(\eta_{\ell}\right) d \sigma(s)
$$

and if $\zeta_{\ell} \in \Sigma(\mathcal{M})$ then $\overline{\zeta_{\ell}} \in \Sigma(\mathcal{M})$ For real characteristic values we have

$$
\xi_{\ell}=\int_{\tau_{\min }}^{\tau_{\max }} \exp \left(\xi_{\ell} s\right) d \sigma(s) \quad\left(\zeta_{\ell}=\xi_{\ell} \in \mathbb{R}\right)
$$


Remark 4.1. Returning to 4.2), the defining equation $\zeta_{\ell}=\int_{\tau_{\min }}^{\tau_{\max }} \exp \left(\zeta_{\ell} s\right) d \sigma(s)$ frequently provides results on the possible size of the characteristic values given conditions on $d \sigma$. To illustrate, consider real characteristic values in in (4.7) and suppose $\tau_{\max }>0$ and $d \sigma(s)$ is of fixed sign (assume it is nonnegative and not everywhere zero) on an interval $\left[\tau_{\max }-\Delta, \tau_{\max }\right]$. Then as $\xi \rightarrow \infty$, the behaviour of $\int_{\tau_{\min }}^{\tau_{\max }} \exp (\xi s) d \sigma(s)$ is dominated by the behaviour of $\int_{\tau_{\max }-\Delta}^{\tau_{\max }} \exp (\xi s) d \sigma(s)$ which grows exponentially, and hence grows faster than $\xi$. Consequently, $\xi<\chi_{\mathcal{L}}(\xi)$ for all large $\xi \in \mathbb{R}$ : $\chi_{\mathcal{M}}(\xi)$ cannot vanish for large $\xi$ and the real characteristic values $\left\{\xi_{\ell}\right\}$ are bounded above.

The multiplicity of a characteristic value $\zeta_{\ell} \in \mathbb{C}$ will be denoted $\mu\left(\zeta_{\ell}\right) \in \mathbb{N}=\{1,2,3, \cdots\}$ :

$$
\chi_{\mathcal{M}}(\zeta)=\chi_{\mathcal{M}}^{\prime}(\zeta)=\cdots=\chi_{\mathcal{M}}^{\mu\left(\zeta_{\ell}\right)-1}(\zeta)=0 \text { and } \chi_{\mathcal{M}}^{\mu\left(\zeta_{\ell}\right)}(\zeta) \neq 0
$$

Specifically for 4.2 , the values of derivatives in 4.8 assume the form

$$
\begin{gathered}
\chi^{\prime}(\zeta)=1-\int_{\tau_{\min }}^{\tau_{\max }} s \exp (\zeta s) d \sigma(s) \\
\chi^{k}(\zeta)=-\int_{\tau_{\min }}^{\tau_{\max }} s^{k} \exp (\zeta s) d \sigma(s) \quad(k=2,3, \cdots) .
\end{gathered}
$$

If $\zeta_{\ell} \in \Sigma(\mathcal{M})$, then $\overline{\zeta_{\ell}} \in \Sigma(\mathcal{M})$ where $\overline{\zeta_{\ell}}$ is the complex conjugate, and $\mu\left(\overline{\zeta_{\ell}}\right)=\mu\left(\zeta_{\ell}\right)$. We refer to a function

$$
v_{\zeta_{\ell}}(t)=P_{\zeta_{\ell}}(t) \exp \left(\zeta_{\ell} t\right) \quad \text { where } P_{\zeta_{\ell}}(t):=\sum_{r=0}^{\mu_{\zeta_{\ell}}-1} \alpha_{\ell, r} t^{r}
$$

as a characteristic solution. Here, $P_{\zeta_{\ell}}(t)$ is an arbitrary polynomial in $t$ of degree $\mu_{\zeta_{\ell}}-1$. When $k \in\left\{1,2, \cdots, \mu_{\zeta_{\ell}}-1\right\}$ and $P_{\zeta_{\ell}}(t)=t^{k}$ we write $v_{\zeta_{\ell}}(t)$ in 4.10 as $v_{\zeta_{\ell}}(k ; t)$.

Lemma 4.1. (a) For $u_{\zeta}(t):=\exp (\zeta t)(\zeta \in \mathbb{C}), u_{\zeta}^{\prime}(t)-\mathcal{L} u_{\zeta}(t)=0$ (i.e., $\mathcal{M} u_{\zeta}(t)=0$ ) for all $t \in \mathbb{R}$ if and only if $\chi_{\mathcal{M}}(\zeta)=0$ (i.e., $\zeta \in \Sigma(\mathcal{M})$ ). (b) Given $\zeta_{\ell} \in \Sigma(\mathcal{M})$, every characteristic solution $v_{\zeta_{\ell}}$ in (4.10) is a (possibly complex-valued) solution of $\mathcal{M} u(t)=0$. (c) When $\zeta_{\ell} \in \Sigma(\mathcal{M})$ has multiplicity $\mu_{\zeta_{\ell}} \geq 1$ and $\xi_{\ell}=\Re\left(\zeta_{\ell}\right), \eta_{\ell}=\Im\left(\zeta_{\ell}\right)$, and $\left\{\alpha_{\ell, r}\right\},\left\{\widehat{\alpha}_{\ell, r}\right\}$ are arbitrary sets of real numbers, we define the corresponding real-valued functions

$$
v_{\zeta_{\ell}}(t)=\sum_{r=0}^{\mu_{\zeta_{\ell}}-1} \alpha_{\ell, r} t^{r} \times \exp \left(\xi_{\ell} t\right) \cos \left(\eta_{\ell} t\right)+\sum_{r=0}^{\mu_{\zeta_{\ell}}-1} \widehat{\alpha}_{\ell, r} t^{r} \times \exp \left(\xi_{\ell} t\right) \sin \left(\eta_{\ell} t\right),
$$

then $\mathcal{M} v_{\zeta_{\ell}}(t)=0$.

Proof: In view of Lemma 3.1 it is sufficient to consider $\mathcal{L} \in \mathfrak{A}_{3.3 .}$. Clearly, for $\zeta \in \mathbb{C}$,

$$
\mathcal{M} \exp (\zeta t) \equiv\{\mathcal{D}-\mathcal{L}\} \exp (\zeta t)=\chi_{\mathcal{M}}(\zeta) \times \exp (\zeta t)
$$

and (a) follows. Now suppose that $\zeta_{\ell} \in \Sigma(\mathcal{M})$ and $\mu\left(\zeta_{\ell}\right) \in \mathbb{N}$ and take $v_{\zeta_{\ell}}(1 ; t)=t \exp \left(\zeta_{\ell} t\right)$. Then

$$
\mathcal{M} v_{\zeta_{\ell}}(1 ; t)=t \exp \left(\zeta_{\ell} t\right) \times \chi_{\mathcal{M}}(\zeta)+\exp \left(\zeta_{\ell} t\right) \times \chi_{\mathcal{M}}^{\prime}(\zeta)=0 \quad(t \in \mathbb{R}) .
$$

Further, if $\mu\left(\zeta_{\ell}\right) \geq 2$, with $k \in\left\{2,3, \cdots, \mu\left(\zeta_{\ell}\right)\right\}, v_{\zeta_{\ell}}(k ; t)=t^{k} \exp \left(\zeta_{\ell} t\right)$. For $t \in \mathbb{R}$,

$$
\mathcal{M} v_{\zeta_{\ell}}(k ; t)=\left\{k t^{(k-1)} \exp \left(\zeta_{\ell} t\right)+\zeta_{\ell} t^{k} \exp \left(\zeta_{\ell} t\right)\right\}-\int_{\tau_{\min }}^{\tau_{\max }}(t+s)^{k} \exp \left\{\zeta_{\ell}(t+s)\right\} d \sigma(s) .
$$

We know that 4.8 and 4.9 hold; for $k \in\left\{2,3, \cdots, \mu\left(\zeta_{\ell}\right)-1\right\}$ (but not for $\left.k=\mu\left(\zeta_{\ell}\right)\right) \int_{\tau_{\min }}^{\tau_{\max }} s^{k} \exp \left\{\zeta_{\ell} s\right\} d \sigma(s)=$ 0 . By the binomial theorem, $(t+s)^{k}=t^{k}+k t^{k-1} s+\cdots+s^{k}$, so for $k \in\left\{2,3, \cdots, \mu\left(\zeta_{\ell}\right)-1\right\}$ we find $\mathcal{M} v_{\zeta_{\ell}}(k ; t)=$

$$
t^{k} \exp \left(\zeta_{\ell} t\right)\left(\zeta_{\ell}-\int_{\tau_{\min }}^{\tau_{\max }} \exp \left\{\zeta_{\ell} s\right\} d \sigma(s)\right)+k t^{(k-1)} \exp \left(\zeta_{\ell} t\right)\left(1-\int_{\tau_{\min }}^{\tau_{\max }}\left\{s \exp \left\{\zeta_{\ell} s\right\} d \sigma(s)\right)\right.
$$


or (as a generalization of 4.13 above)

$$
\mathcal{M} v_{\zeta_{\ell}}(k ; t)=t^{k} \exp \left(\zeta_{\ell} t\right) \times \chi_{\mathcal{M}}\left(\zeta_{\ell}\right)+k t^{(k-1)} \exp \left(\zeta_{\ell} t\right) \times \chi_{\mathcal{M}}^{\prime}\left(\zeta_{\ell}\right),
$$

which vanishes for every $t$. By superposition, we establish that 4.10 is a solution and (b) follows. Part (c) follows by considering the real and imaginary parts of (4.10).

Suppose $\left\{\alpha_{\ell, r}\right\},\left\{\widehat{\alpha}_{\ell, r}\right\}$ in 4.11 are such that

$$
v(t):=\sum_{\zeta_{\ell} \in \Sigma(\mathcal{M})}\left\{v_{\zeta_{\ell}}(t)\right\}
$$

converges for all $t \geq 0$. For convenience (see Lemma 4.2, we suppose sup $\left|\alpha_{\ell, r}\right| \leq \Omega<\infty$ and $\sup \left|\widehat{\alpha}_{\ell, r}\right| \leq \Omega<\infty$. Proceeding formally $\mathcal{M}\left(\sum_{\zeta_{\ell} \in \Sigma(\mathcal{M})}\left\{v_{\zeta_{\ell}}(t)\right\}\right)=\sum_{\zeta_{\ell} \in \Sigma(\mathcal{M})} \mathcal{M}\left\{v_{\zeta_{\ell}}\right\}(t)$. and very term in the right-hand sum vanishes. However, without preciser assumptions there is no guarantee that every function $v(t)$ that satisfies $\mathcal{M} v(t)=0$ can be written in the form 4.15.

Remark 4.2 (Term-by-term differentiation and integration). With arbitrary parameters in (4.10 there is no guarantee that either series converges. As noted in [6] there are series of continuouslydifferentiable functions, uniformly convergent on an interval, for which the series obtained by termby-term differentiation converges on the interval, but the sum of the original series is either not differentiable on the whole interval in question, or it is differentiable but its derivative is not equal to the sum of the series of derivatives.

Let $\mathfrak{T} \equiv\left[T_{\min }, T_{\max }\right] \subset \mathbb{R}$ and consider condition $\$^{2}$ for term-by-term differentiation of a series $S=\sum_{\ell=1}^{\infty} w_{\ell}(t)$. If the terms $w_{\ell}(t)$ are continuously differentiable on the interval $\mathfrak{T}$, if the infinite series $S$ converges at some point $t_{0}$ of $\mathfrak{T}$ and the series $\sum_{\ell=1}^{\infty} w_{\ell}^{\prime}(t)$ of derivatives of the terms is uniformly convergent on $\mathfrak{T}$, then the series $S$ is itself uniformly convergent on $\mathfrak{T}$, its sum is continuously differentiable on $\mathfrak{T}$ and $S^{\prime}(t)$ can be obtained by term-by-term differentiation: $S^{\prime}(t)=$ $\sum_{\ell=1}^{\infty} w_{\ell}^{\prime}(t)$ for $t \in \mathfrak{T}$. The conditions above may be applied with $w_{\ell}=v_{\zeta_{\ell}}$.

\section{Solution properties}

We summarize the position. A real-valued uniformly convergent series

$$
\sum_{\zeta_{\ell} \in \Sigma(\mathcal{M})}\left\{\left(\sum_{r=0}^{\mu_{\zeta_{\ell}}-1} a_{\ell, r} t^{r}\right) \exp \left(\zeta_{\ell} t\right)\right\}
$$

of characteristic solutions $v_{\zeta_{\ell}}(t)$ is a (possibly complex-valued) solution of $\mathcal{M} u(t)=0$ for $t \in \mathfrak{T}$. The real and imaginary parts of solutions of the form (5.1) are real-valued solutions, related to linear combinations of solutions of the form 4.11. From Lemma 4.1 and with its notation, we have (by superposition, and Remark 4.2 the following result.

Theorem 5.1. Every uniformly convergent series on $\mathfrak{T} \subset \mathbb{R}$ of the form

$$
\begin{gathered}
\sum_{\ell=1}^{\infty} \sum_{r=0}^{\mu_{\zeta_{\ell}}-1}\left\{\alpha_{\ell, r} t^{r} \times \cos \left(\eta_{\ell} t\right)+\widehat{\alpha}_{\ell, r} t^{r} \times \sin \left(\eta_{\ell} t\right)\right\} \exp \left(\xi_{\ell} t\right), \\
\xi_{\ell}=\Re\left(\zeta_{\ell}\right), \eta_{\ell}=\Im\left(\zeta_{\ell}\right), \quad \zeta_{\ell} \in \Sigma(\mathcal{M}),
\end{gathered}
$$

(in particular, sums over a finite number of terms $\ell \in\{1,2, \cdots, N\}$ ), satisfies $\mathcal{M} u(t)=0$ with $\mathcal{M}=\mathcal{D}-\mathcal{L}$, and $\mathcal{L} \in \mathfrak{A}_{\sqrt[3.3]{ },}$, when $\left[t+\tau_{\min }, t+\tau_{\max }\right] \subset \mathfrak{T} \subset \mathbb{R}$.

\footnotetext{
${ }^{2}$ See Uniformly-convergent series, Encyclopedia of Mathematics, [6]
} 
The expression in (5.2) contains arbitrary parameters $\left\{\alpha_{\ell, r}\right\},\left\{\widehat{\alpha}_{\ell, r}\right\}$, and if a particular solution is to be defined then suitable constrains (e.g., initial conditions, final conditions, or similar) would have to be specified. To study this issue would take us into the area of problems that are well- or ill-posed in the sense of Hadamard, an area into which there is no need for us to venture here.

In applications, various properties (e.g., periodicity, oscillation or non-oscillation, decay, boundedness or unboundedness) of solutions to $\mathcal{M} u(t)=0$ are of interest. Lemma 4.1 provides insight because it establishes certain forms of solution, but we have not established that all solutions of $\mathcal{M} u(t)=0$ have the form 5.2 . It may be sufficient in our discussion to establish that all solutions possesing a specific qualitative property can be written in the form (5.2).

By a periodic solution with period $\varpi \in \mathbb{R}$ we mean a solution that satisfies $u(t)=u(t+\varpi)$ for $t \in \mathbb{R}$, and such solutions provide examples of oscillatory functions: In normal parlance, these are functions that vary repeatedly about a central value. For $A \neq 0$,

$$
1-A \exp (\xi t) \cos (\eta t) \quad(t \in \mathbb{R})
$$

oscillates about 1 with steady amplitude if $\xi=0$ and with increasing or decreasing amplitude as $t \rightarrow \infty$, according as $\xi>0$ or $\xi<0$. A different definition of oscillation is often employed in the discussion of solutions of differential equations and it has been carried across to equations of Class D, A, or M (Definition 3.4

Definition 5.1. Here, a non-null real-valued solution will be said to be ultimately oscillating if it has an unbounded sequence of distinct real zeros.

The function (5.3) is not "ultimately oscillating" in the sense of Definition 5.1 unless $\eta \neq 0$ and either $\xi>0$ or $\xi=0$ and $A=1$.

\subsection{A general perspective}

Equation $(\star)$ in the abstract reads:

$$
u^{\prime}(t)=\int_{-\tau}^{\tau} u(t+s) d \sigma(s) \quad(t \in \mathbb{R})
$$

and is defined by a real-valued integrator $\sigma$, which is independent of $v$, and is of bounded variation. The integral is a Riemann-Stieltjes integral. By a solution $u$ of $(5.4)$ we mean a real-valued function ${ }^{3}$ $u$ that is differentiable and satisfies (5.4). Equation (2.1) can be expressed in the form (5.4) (with normalized $\tau=1$ ), and the study of (2.1) will find persistent echoes in what follows. However, it is not universally the case that all solutions of equations of the type $(5.4)$ are expressible in the form (2.4) (cf. (7.13) ), and we shall impose conditions to overcome this difficulty.

The symmetry about $t$ of the deviating arguments $t \pm 1$ in (2.1) makes the example (2.1), and many others in the literature, somewhat special. We wish to explore examples that lack this symmetry e.g., $u^{\prime}(t)=\frac{1}{2}\left\{u\left(t+\frac{3}{2}\right)-u\left(t-\frac{1}{2}\right)\right\}, t \in \mathbb{R}$. Equation (5.4) appears to display the symmetry referred to here, but if the support of $\sigma$ is $\left[\tau_{\natural}, \tau^{\natural}\right]$ with $\tau_{\natural}<\tau^{\natural}$ then we can rewrite (5.4) in an explicitly unsymmetric form

$$
u^{\prime}(t)=\int_{\tau_{\natural}}^{\tau^{\natural}} u(t+s) d \sigma(s) \quad(t \in \mathbb{R}) .
$$

Remark 5.1 (Equivalent versions of (5.4) and (5.5)). Equation (5.4) is a special case of (5.5) (and vice-versa). We can replace $\left[\tau_{\natural}, \tau^{\natural}\right]$ by $[-\tau, \tau]$ assuming (as we do) that we permit d $\sigma(s)$ to vanish on subsets of $[-\tau, \tau]$. For an equivalent form (5.4) of (5.5) we take

$$
\tau:=\max \left\{\tau_{\natural}, \tau^{\natural}\right\}, \quad d \sigma(s)=0 \text { if } s \notin\left[-\tau_{\natural}, \tau^{\natural}\right] .
$$

\footnotetext{
${ }^{3}$ There is a natural extension to complex-valued functions which we refer to as solutions if their real and their imaginary parts are both solutions.
} 
Definition 5.2 (Delayed, advanced, and mixed-type equations). Equation (5.5) and (5.4) are ordinary differential equations if $d \sigma(s)=0$ for $s \neq 0$. Equation (5.5) (and (5.4) are, if not ordinary differential equations, said to be

(i) of retarded or delayed type if $\tau^{\natural}=0(d \sigma(s)=0$ for $s>0$ in (5.4) $)$,

(ii) of advanced type if $\tau_{\natural}=0(d \sigma(s)=0$ for $s<0$ in $(5.4)$ ), and

(iii) of mixed type if it is neither of retarded nor advanced type.

Where $\tau>0$, the equations $u^{\prime}(t)=a u(t)+b u(t-\tau)+c u(t+\tau)$ illustrate the above types, either with $b$ and $c=0$ or alternatively with (i) $c=0$, or (ii) with $b=0$, or (iii) $b c \neq 0$.

\subsection{Solution properties considered in our discussion}

Our properties or interest are boundedness and oscillation. A solution $u$ is bounded for $t \geq 0$ if $\sup _{t>0}|u(t)|<\infty$. Our discussion of boundedness of all solutions for $t \geq 0$ reduces, under our assumptions, to an investigation of the existence or non-existence of zeros of the characteristic function that have positive real part.

In Definition 7.1 we define oscillatory and non-oscillatory solutions precisely, and then use these definitions to define non-oscillatory and oscillatory equations. Using Definition 7.3 and Definition 7.5), our discussion of oscillation reduces, under our assumptions, to an investigation of the existence or non-existence of real zeros of $\chi$.

As the zeros of $\chi$ lie in the complex plane, it has been conventional for investigations of the characteristic values to use complex analysis, but we can obtain some insight very readily using real analysis.

\section{Linear homogeneous autonomous equations with deviating arguments}

We next review types of functional differential equations that can be written in the form

$$
\mathcal{M} u(t)=0 \text { where } \mathcal{M} v(t):=v^{\prime}(t)-\int_{-\tau}^{\tau} v(t+s) d \sigma(s),
$$

for differentiable $v \in([-\tau, \tau] \rightarrow \mathbb{R})$. It is natural to seek appropriate "canonical equations" that may be used as "paradigms" when assessing the qualitative behaviour of solutions. There seems to be some lack of uniformity in the explicit forms of the equations chosen for study in the literature, and we consider the refined version of 6.1,

$$
\mathcal{M}_{\natural} u(t)=0 \text { where } \mathcal{M}_{\natural} v(t):=v^{\prime}(t)-\int_{-\tau_{\natural}}^{\tau^{\natural}} v(t+s) d \sigma(s) \quad(t \in \mathbb{R}) .
$$

We write

$$
\mathcal{M}_{\natural} v(t):=v^{\prime}(t)-\mathcal{L}_{\natural} v(t) \equiv \mathcal{D} v(t)-\mathcal{L}_{\natural} v(t)
$$

for differentiable functions $v \in\left(\left[-\tau_{\natural}, \tau^{\natural}\right] \rightarrow \mathbb{R}\right)$, with

$$
\mathcal{D} v(t):=v^{\prime}(t) \text { and } \mathcal{L}_{\natural} v(t):=\int_{-\tau_{\natural}}^{\tau^{\natural}} v(t+s) d \sigma(s) \quad(\text { for } t \in \mathbb{R}) \text {. }
$$

We regard $\mathcal{L}_{\natural}$ as an operator on the space $C\left(\left[\tau_{\natural}, \tau^{\natural}\right] \rightarrow \mathbb{R}\right)$ of continuous real-valued functions and $\mathcal{M}_{\natural}$ as an operator on the space of differentiable real-valued functions on $\left[\tau_{\natural}, \tau^{\natural}\right]$, with supremum norm ( $\mathcal{D}$ is an unbounded operator on this space). The equation $\mathcal{M}_{\natural} u(t)=0$ in 6.2 now defines a wide class of autonomous linear differential equations.

Definition 6.1 (Real or complex-valued functions). Suppose that $v \in C(\mathbb{R} \rightarrow \mathbb{R})$. We use the notation $\mathcal{L}_{\natural}\{v(t)\}$ as a synonym for $\mathcal{L}_{\natural} v(t)$ and $\mathcal{M}_{\natural}\{v(t)\}$ as a synonym for $\mathcal{M}_{\natural} v(t)$ when $v$ is differentiable. Now if $v$ is a complex-valued function $\left(v \in C(\mathbb{R} \rightarrow \mathbb{C})\right.$, with $v_{1}(t)=\Re\{v(t)\}$ and $v_{2}(t)=\Im\{v(t)\}$ for $\left.t \in \mathbb{R}\right), \mathcal{M}_{\sharp}\{v(t)\}$ will denote $\mathcal{M}_{\sharp}\left\{v_{1}(t)\right\}+\mathrm{i} \mathcal{M}_{\natural}\left\{v_{2}(t)\right\}$. 
Remark 6.1. Some authors consider equations $\mathcal{M}_{\natural} u(t)=0$ that hold only for $t \in[-\tau, \infty)$. Any solution for $t \in \mathbb{R}$ is obviously a solution in the sense of these authors (i.e., "on the half-line $[-\tau, \infty)$ "). In our work, we are interested in the properties of solutions for $t \geq 0$, in particular as $t \rightarrow+\infty$.

In (6.3), the function $v$ is to be differentiable, and $\sigma$ is of bounded variation. Suppose that $\sigma$ is a (so-called staircase) step function with a finite number of jumps at the points $\left\{s_{j}\right\}$. Then 6.3b assumes the form $\mathcal{L}_{\natural} v(t):=\sum_{j} \gamma_{j} v\left(t+s_{j}\right)$, where $-\tau_{\natural} \leq s_{j} \leq \tau^{\natural}$ for all $j$. Alternatively, suppose that $\sigma$ is absolutely continuous. Then $6.3 \mathrm{~b}$ assumes the form $\mathcal{L}_{\natural} v(t):=\int_{-\tau_{\natural}}^{\tau^{\natural}} v(t+s) \gamma(s) d(s)$, with $\gamma(s)=\sigma^{\prime}(s)$. If $\sigma$ decomposes as the sum of such a discontinuous part and an absolutely continuous part then

$$
\mathcal{M}_{\natural} v(t)=v^{\prime}(t)-\mathcal{L}_{\natural} v(t) \text { and } \mathcal{L}_{\natural} v(t):=\sum_{j} \gamma_{j} v\left(t+s_{j}\right)+\int_{-\tau_{\natural}}^{\tau^{\natural}} v(t+s) \gamma(s) d(s) .
$$

When $u$ is a solution it is supposed differentiable; however, $v$ does not have to be differentiable (nor even continuous) for the expression for $\mathcal{L}_{\natural} v(t)$ on the right of $(6.4)$ to have a meaning - we only require the sum and Riemann integral to be defined.

Example 6.1. The equation that corresponds to 6.4 reads

$$
u^{\prime}(t)=\sum_{j} \gamma_{j} u\left(t+s_{j}\right)+\int_{-\tau_{\natural}}^{\tau^{\natural}} u(t+s) \gamma(s) d(s) .
$$

We use examples of 6.5) to illustrate the classes of equation in Definition 5.2. Suppose $\tau_{0}, \tau^{0}$, and $\tau_{ \pm k}$ non-negative, $\beta$ and $\gamma$ are non-trivial, and $\sum_{0}^{K}\left\{\left|b_{k}\right|+\left|c_{k}\right|\right\} \neq 0$. Consider

$$
u^{\prime}(t)=a u(t)+\sum_{k=1}^{K} b_{k} u\left(t-\tau_{k}\right)+\sum_{k=1}^{K} c_{k} u\left(t+\tau^{k}\right)+b_{0} \int_{-\tau_{0}}^{0} u(t+s) \beta(s) d s+c_{0} \int_{0}^{\tau^{0}} u(t+s) \gamma(s) d s .
$$

This equation is of the type shown in 6.5); it is (i) of advanced type if $b_{k}=0$ for all $k \in$ $\{0,1,2, \cdots, K\}$ and (ii) of retarded type if $c_{k}=0$ for all $k \in\{0,1,2, \cdots, K\}$.

\section{Qualitative behaviour of solutions}

Much of the literature relating to the qualitative behaviour of solutions of functional differential equations is concerned with the asymptotic behaviour of solutions. As we remarked earlier, this involves investigating the existence (or otherwise) of characteristic values with positive real partsee, for example, 2, 7], see also [1] for further background. Less common is the approach we adopt here, which focuses on oscillatory behaviour.

\subsection{Definitions related to Oscillation $\mathcal{E}$ Non-oscillation}

The literature includes, for a variety of equations expressible in the form $(6.3)$, studies on methods for determining whether some of the solutions of such an equation are non-oscillatory or all are oscillatory, and a rôle for the corresponding characteristic function in the study of this issue is documented. We refine existing definitions and results and discuss the choice of canonical equations. First we consider again the definition of oscillatory function:

Definition 7.1 (Non-oscillation and oscillation of a function). A real-valued function of a real variable, $x \in(\mathbb{R} \rightarrow \mathbb{R})$, is said to be non-oscillatory - to be precise, non-oscillatory about zero for increasing argument - when there exists $T<\infty$ such that

$$
\operatorname{sign}\left\{u\left(t_{1}\right)\right\} \times \operatorname{sign}\left\{u\left(t_{2}\right)\right\} \neq-1 \text { for all } t_{1}, t_{2} \geq T .
$$

When a function is not non-oscillatory (as above) then it is called oscillatory (about zero, for increasing argument). 
Remark 7.1. (a) The words "for increasing argument" in Definition 7.1 are in general omitted as also, with less justification, are the words "about zero". (b) According to Definition 7.2. for the null function $v_{0}$ (with $v_{0}(t) \equiv 0$ ), $\operatorname{sign}\left\{v_{0}\left(t_{1}^{\prime}\right)\right\}=\operatorname{sign}\left\{v_{0}\left(t_{2}^{\prime}\right)\right\}=0$ for all $t_{1}^{\prime}, t_{2}^{\prime}$ by our definition of the sign function. Thus, the function $u_{0}$ that vanishes everywhere is oscillatory.

To cover behaviour as $t \rightarrow-\infty$, where solutions are defined for negative arguments, we say that a function $v$, whose domain includes $(-\infty, 0]$, is non-oscillatory for decreasing argument if the function $v_{-}$with $v_{-}(t)=v(-t)$ for $t \in \mathbb{R}$ is non-oscillatory for increasing argument, and oscillatory for decreasing argument if the function $v_{-}$is oscillatory for increasing argument.

7.2. Properties of the characteristic roots: Cases with $\operatorname{sign}\left\{\lim _{x \rightarrow-\infty} \chi_{\mathcal{M}_{\sharp}}(x)\right\}=-\operatorname{sign}\left\{\lim _{x \rightarrow+\infty} \chi_{\mathcal{M}_{\sharp}}(x)\right\}$

The meaning of the sign of a number is not universally accepted, so we state the definition that we use:

Definition 7.2 (The sign of a finite or infinite real number $w$ ). We define

$\operatorname{sign}(-\infty)=-1, \operatorname{sign}(0)=0, \operatorname{sign}(+\infty)=+1, \operatorname{sign}(w)=w /|w|$ otherwise, for $w \in \mathbb{R}$.

Theorem 7.1 (Sufficient conditions for a real characteristic value). The characteristic function $\chi_{\mathcal{M}_{\sharp}}$ has at least one real zero if and only if

$$
\operatorname{sign}\left\{\inf _{x \in \mathbb{R}} \chi_{\mathcal{M}_{\sharp}}(x)\right\} \times \operatorname{sign}\left\{\sup _{x \in \mathbb{R}} \chi_{\mathcal{M}_{\sharp}}(x)\right\} \in\{-1,0\} .
$$

Further, the characteristic function $\chi_{\mathcal{M}_{\mathfrak{h}}}$ has an odd number of real zeros (counting according to multiplicity) if and only if $\operatorname{sign}\left\{\lim _{x \rightarrow-\infty} \chi_{\mathcal{M}_{\sharp}}(x)\right\}=-\operatorname{sign}\left\{\lim _{x \rightarrow+\infty} \chi_{\mathcal{M}_{\sharp}}(x)\right\}$.

[Proof: : Follows immediately from the continuity of $\chi_{\mathcal{M}_{\natural}}$ ]

Example 7.1. Consider the case $\mathcal{M}_{\natural} u(t)=u^{\prime}(t)-a u\left(t-\tau_{1}\right)-b u(t)-c u\left(t+\tau_{2}\right)$ with $\tau_{1,2}>0$, for which $\chi_{\mathcal{M}_{\natural}}(\zeta)=\zeta-a \exp \left(-\tau_{1} \zeta\right)-b \exp (\zeta)-c \exp \left(+\tau_{2} \zeta\right)$ when $\zeta \in \mathbb{C}$ and

$$
\chi_{\mathcal{M}_{\natural}}(x)=x-a \exp \left(-\tau_{1} x\right)-b \exp (x)-c \exp \left(+\tau_{2} x\right) \text { where } x \in \mathbb{R} .
$$

It can be verified that $\mathcal{M}_{\sharp} \in \mathfrak{L}^{\sharp}$. Now

$$
\operatorname{sign}\left\{\lim _{x \rightarrow-\infty} \chi_{\mathcal{M}_{\sharp}}(x)\right\}=-1 \text { if } a \geq 0 ; \quad \operatorname{sign}\left\{\lim _{x \rightarrow-\infty} \chi_{\mathcal{M}_{\sharp}}(x)\right\}=+1 \text { if } a<0 ;
$$

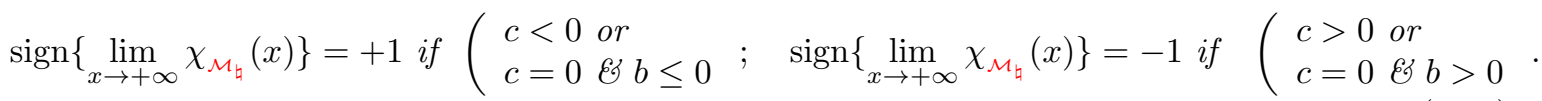

Then (7.3) holds in the following cases:

$$
\begin{array}{lll}
a \geq 0 & \mathscr{G} & \{c<0, \text { or } c=0 \quad \mathcal{G} b \leq 0\} \\
a<0 & \mathscr{G} \quad\{c>0, \text { or } c=0 \quad \mathcal{G} b>0\}
\end{array}
$$

7.3. A case with $\operatorname{sign}\left\{\lim _{x \rightarrow-\infty} \chi_{\mathcal{M}_{\sharp}}(x)\right\}=\operatorname{sign}\left\{\lim _{x \rightarrow+\infty} \chi_{\mathcal{M}_{\sharp}}(x)\right\}$

Consider a case in which $\operatorname{sign}\left\{\lim _{x \rightarrow-\infty} \chi_{\mathcal{M}_{\sharp}}(x)\right\} \neq-\operatorname{sign}\left\{\lim _{x \rightarrow+\infty} \chi_{\mathcal{M}_{\sharp}}(x)\right\}$ so that Proposition 7.1 does not apply. To develop the discussion, impose the assumption that $\chi_{\mathcal{M}_{\natural}}$ is concave either downwards or upwards, in a strict sense. (E.g., if $\chi_{\mathcal{M}_{\natural}}$ is strictly concave downwards $\chi_{\mathcal{M}_{\sharp}}\left(\lambda x_{1}+\right.$ $\left.(1-\lambda) x_{2}\right)>\lambda \chi_{\mathcal{M}_{\sharp}}\left(x_{1}\right)+(1-\lambda) \chi_{\mathcal{M}_{\sharp}}\left(x_{2}\right)$ for $x_{1} \neq x_{2}, \lambda \in(0,1)$, and $\lim _{x \rightarrow \pm \infty} \chi_{\mathcal{M}_{\sharp}}(x)=-\infty$.) Thus,

$$
\text { either } \chi_{\mathcal{M}_{\sharp}}^{\prime \prime}(x)<0 \text { for all } x \in \mathbb{R} \text { or } \chi_{\mathcal{M}_{\sharp}}^{\prime \prime}(x)>0 \text { for all } x \in \mathbb{R} \text {. }
$$


Equivalently, since $\chi_{\mathcal{M}_{\sharp}}(x)=x-\omega_{\mathcal{L}_{\sharp}}(x)(x \in \mathbb{R})$,

$$
\text { either } \omega_{\mathcal{L}_{\sharp}}{ }^{\prime \prime}(x)>0 \text { for all } x \in \mathbb{R} \quad \text { or } \quad \omega_{\mathcal{L}_{\sharp}}{ }^{\prime \prime}(x)<0 \text { for all } x \in \mathbb{R}
$$

and $\omega_{\mathcal{L}_{\natural}}$ is strictly concave (upwards or downwards). In the first case (also called "convex"):

$$
\omega_{\mathcal{L}_{\sharp}}\left(\lambda x_{1}+(1-\lambda) x_{2}\right)<\lambda \omega_{\mathcal{L}_{\sharp}}\left(x_{1}\right)+(1-\lambda) \omega_{\mathcal{L}_{\sharp}}\left(x_{2}\right)\left(\text { when } x_{1} \neq x_{2} \text { and } \lambda \in(0,1)\right.
$$

and $\operatorname{sign}\left\{\lim _{x \rightarrow-\infty} \omega_{\mathcal{L}_{\natural}}(x)\right\}=\operatorname{sign}\left\{\lim _{x \rightarrow+\infty} \omega_{\mathcal{L}_{\natural}}(x)\right\}=1$ while, in the second case, $\operatorname{sign}\left\{\lim _{x \rightarrow-\infty} \omega_{\mathcal{L}_{\natural}}(x)\right\}=$ $\operatorname{sign}\left\{\lim _{x \rightarrow+\infty} \omega_{\mathcal{L}_{\natural}}(x)\right\}=-1$.

Theorem 7.2. Given the characteristic function $\chi_{\mathcal{M}_{\sharp}}(x)=x-\omega_{\mathcal{L}_{\sharp}}(x)$, suppose that the analytic function $\omega_{\mathcal{L}_{\natural}}$ is concave on $\mathbb{R}$. Then one of the following possibilities holds (i) $\chi_{\mathcal{M}_{\natural}}$ has no real zeros; (ii) $\chi_{\mathcal{M}_{\sharp}}$ has one zero $\zeta^{\natural}$ where $\chi_{\mathcal{M}_{\natural}}^{\prime}\left(\zeta^{\natural}\right)=0$; (iii) $\chi_{\mathcal{M}_{\natural}}$ has two distinct real zeros $\zeta^{\natural} 1$ and $\zeta^{\natural}$.

[Proof: For definiteness suppose that $\omega_{\mathcal{L}_{\natural}}$ is concave upwards (and a line segment between two points on the graph lies above the graph connecting the two points). Real zeros of $\omega_{\mathcal{L}_{\natural}}$ arise if $x=\omega_{\mathcal{L}_{\natural}}(x)$ for some $x \in \mathbb{R}$. The case (i) arises when $x<\omega_{\mathcal{L}_{\natural}}(x)$ for all $x \in \mathbb{R}$; the case (ii) arises when the line $y=x$ is tangential to the curve $y=\omega_{\mathcal{L}_{\natural}}(x)$ and there are then "two coincident zeros"; for case (iii) the line $y=x$ intersects the curve $y=\omega_{\mathcal{L}_{\natural}}(x)$ in two distinct points and there are two distinct real zeros of $\chi_{\mathcal{M}_{\sharp}}$. The case where $\omega_{\mathcal{L}_{\sharp}}$ is concave downwards is analogous.]

Example 7.2. Consider the case

$$
\mathcal{M}_{\natural} u(t)=u^{\prime}(t)-b_{1} u\left(t-\tau_{1}\right)-a u(t)-c_{1} u\left(t+\tau_{1}\right) \text { with } \tau_{1}>0
$$

(a simplified version of 6.6), for which

$$
\chi_{\mathcal{M}_{\sharp}}(x)=x-b_{1} \exp \left(-\tau_{1} x\right)-a-c_{1} \exp \left(+\tau_{1} x\right)
$$

can be written, when $x$ is real, as

$$
\begin{gathered}
\chi_{\mathcal{M}_{\sharp}}(x)=x-a_{0}-A \sinh \left(\tau_{1} x\right)-B \cosh \left(\tau_{1} x\right) \text { for } x \in \mathbb{R} . \\
A=c_{1}-b_{1}, B=b_{1}+c_{1} .
\end{gathered}
$$

We observe the following:

1. If $A=0$ and $B>0$ (i.e., $c_{1}=b_{1}>0$ ) then $\omega_{\mathcal{L}_{\sharp}}(x)=B \cosh \left(\tau_{1} x\right)$ so $\omega_{\mathcal{L}_{\natural}}$ is concave upwards and Proposition 7.2 holds.

2. If $A=0$ and $B<0$, then $\omega_{\mathcal{L}_{\natural}}$ is concave downwards.

3. On the other hand, if $B=0$ then $\chi_{\mathcal{M}_{\mathfrak{b}}}(x)=x-a-A \sinh \left(\tau_{1} x\right)$ and Proposition 7.1 applies for every $A \in \mathbb{R}$.

If $A=0$ (i.e., whenever $c_{1}=b_{1}$ ) the deviating arguments, with values $t \pm \tau_{1}$, are symmetrically placed about $t$ and are given equal weight in the expression $\mathcal{M}_{\natural} u(t)$.

\subsection{Equations that are oscillatory or non-oscillatory}

We employ the following terminology.

Definition 7.3 (Equations that are oscillatory or non-oscillatory). A scalar homogeneous and autonomous equation $\mathcal{M}_{\natural} u(t)=0$ is called oscillatory (for increasing argument) if all of its solutions are oscillatory for increasing argument, in the sense of Definition 7.1 - otherwise, it is called non-oscillatory (for increasing argument).

\footnotetext{
${ }^{4}$ Other definitions can be found in the literature; some apply to functions defined on a half-line; some definitions apply to vector-valued functions.
} 
The corresponding definitions "for decreasing arguments" (when solutions are defined on the whole set $\mathbb{R}$ ) should be inferred. According to Definition 7.3 the class of non-oscillatory equations $\mathcal{M}_{\natural} u(t)=0$ consists of those equations that have at least one solution that is non-oscillatory for increasing argument.

Every equation $\mathcal{M}_{\natural} u(t)=0$ has the null function $u(t) \equiv 0$ amongst its solutions. In consequence, no equation $\mathcal{M}_{\natural} u(t)=0$ would be oscillatory unless we regard the null function as oscillatory, and Definition 7.1 ensures that this is the case. An alternative approach would be to modify Definition 7.3 by requiring $\mathcal{M}_{\natural} u(t)=0$ to be called oscillatory if all of its non-zero solutions are oscillatory.

\subsection{Criteria involving the characteristic function}

It is clear that if $\chi_{\mathcal{M}_{\natural}}$ has a zero $\zeta_{\natural} \in \mathbb{C}$ with non-zero imaginary part then the equation $\mathcal{M}_{\natural} u(t)=$ 0 possesses some oscillatory solutions since at least one of the characteristic solutions is oscillatory both for increasing argument and for decreasing argument). What is not immediately obvious is whether none of the solutions are oscillatory if all the zeros of the characteristic function $\chi_{\mathcal{M}_{\sharp}}$ are real numbers.

It is apparent from the literature that the direct correlation between oscillatory solutions and the zeros of the characteristic function holds for certain classes of equations but not for all. Krisztin [10] (see also [8, 9]) gives an example showing that (5.4) may have a nonoscillatory solution in spite of the fact that the corresponding characteristic equation has no real roots. The root of the difficulty can be identified if we introduce the following definitions.

Definition 7.4 (Expansions in weighted sums of characteristic functions). and the class $\mathfrak{E}_{\mathcal{M}_{\sharp}}$

Suppose that $\left\{\xi_{\ell}\right\}_{0}^{\infty}$ are the characteristic values of $\mathcal{L}_{\natural}$ and $\xi_{\ell}$ is a characteristic value of finite multiplicity $n_{\ell}$ :

$$
\chi_{\mathcal{M}_{\sharp}}\left(\xi_{\ell}\right)=\chi_{\mathcal{M}_{\natural}}^{\prime}\left(\xi_{\ell}\right)=\cdots=\chi_{\mathcal{M}_{\natural}}^{n_{\ell}-1}\left(\xi_{\ell}\right)=0 \quad(\text { for } \ell \in\{0,1,2, \cdots\}), \quad \chi_{\mathcal{M}_{\natural}}^{n_{\ell}}\left(\xi_{\ell}\right) \neq 0 .
$$

A solution $u$ of the equation $\mathcal{M}_{\natural}\{u\}(t)=0(t \in \mathbb{R})$ is said to be expandable in weighted characteristic functions of $\mathcal{L}_{\natural}$ if it can be expressed

$$
u(t)=\sum_{\ell=0}^{\infty} a_{\ell} \Omega_{\ell}(t) \text { for } t \geq 0
$$

where the functions $\left\{\Omega_{\ell}(t)\right\}$ have the form $\Omega_{\ell}(t)=P_{\ell}(t) \exp \left(\xi_{\ell} t\right)$, in which $P_{\ell}$ is some polynomial of degree $n_{\ell}-1$. If 7.13 holds, we write $u \in \mathfrak{E}_{\mathcal{M}_{\natural}}$.

In the above, the functions $\left\{\Omega_{\ell}\right\}$ are polynomial multiples of characteristic functions.

The class $\mathfrak{E}_{\mathcal{M}^{\natural}}$ is related to the class of exponentially bounded functions ${ }^{5}$. A real-valued function $v$ whose domain includes the positive real numbers is exponentially bounded if there exist $\kappa>0$ and $\alpha \in \mathbb{R}$ such that $\sup _{t \geq 0}|v(t)| \leq \kappa \exp (\alpha t)$.

Definition 7.5 (Oscillatory \& non-oscillatory equations in a weak sense). A homogeneous autonomous scalar equation $\mathcal{M}_{\natural} u(t)=0$ is called non-oscillatory in a weak sense if all of the solutions $u$ contained in $\mathfrak{E}_{\mathcal{M}^{\natural}}$ are non-oscillatory for increasing argument, in the sense of Definition 7.1: - otherwise, it is called oscillatory in a weak sense.

Proposition 7.1. The equation $\mathcal{M}_{\natural} u(t)=0$ is non-oscillatory in a weak sense (for increasing argument) if it is non-oscillatory (for increasing argument) in the sense of Definition 7.3 .

\footnotetext{
${ }^{5}$ For a class of equations discussed in [10, Krisztin establishes that all "non-oscillatory solutions" are exponentially bounded.
} 


\subsection{The class $\mathfrak{L}^{\sharp}$ of operators $\mathcal{L}_{\sharp}$}

For a given family of operators $\mathcal{L}_{\downarrow}$, a question of interest is whether the term "non-oscillatory" and "non-oscillatory in a weak sense" mean the same thing. We introduce the class $\mathfrak{L} \sharp$ of operators $\mathcal{L}_{\natural}$ to avoid this uncertainty. We later provide a refinement (see Definition 7.7) in which we define a class $\mathfrak{L} \supseteq \mathfrak{L}^{\sharp}$.

Definition 7.6 (The class $\mathfrak{L}^{\sharp}$ of operators $\mathcal{L}_{\natural}$ ). We state that $\mathcal{M}_{\natural} \in \mathfrak{L}^{\sharp}$ if the equation $\mathcal{M}_{\natural} u(t)=$ 0 is oscillatory when and only when all the characteristic values have non-zero imaginary parts.

Assumption 7.1. We assume that $\mathcal{M}_{\sharp} \in \mathfrak{L}^{\sharp}$.

Remark 7.2. The literature contains necessary or sufficient results for validity of Assumption 7.1 in the case of equations $\mathcal{L}_{\mathfrak{t}} u(t)=0$ that have only retarded arguments. For mixed equations, Krisztin [10, Assumption H] has given a sufficient condition for Assumption 7.1 to be valid. In the original source, Krisztin took $[-\tau, \tau]$ to be $[-1,1]$ and considers systems of equations. He states a more complex condition and deduces the results stated below as corollaries. When applied to the scalar equation $\mathcal{M}_{\natural} u(t)=0$ with $\mathcal{M}_{\natural}$ as in (6.3) his analysis yields results stated here.

Unless stated otherwise, Assumption 7.1 is taken as given, but a useful alternative approach follows on using the next definition.

Definition 7.7 (The class $\mathfrak{L}$ of operators $\mathcal{L}_{\natural}$ ). We write $\mathcal{M}_{\natural} \in \mathfrak{L}$ if a necessary and sufficient condition for $\mathcal{M}_{\natural} u(t)=0$ to be oscillatory in the weak sense is that all characteristic values have non-zero imaginary parts; equivalently no zeros of the characteristic function $\chi_{\mathcal{M}_{\natural}}$ are purely real.

\subsection{Existing results on $\mathfrak{L}^{\sharp}$}

Proposition 7.2 (A condition from the work [10] of Krisztin). Suppose $\sigma$ defines $\mathcal{M}_{\sharp}$ in 6.3 . Assumption 7.1 is valid when $\sigma$ has an atom at $\tau$, i.e., $\sigma$ satisfies the condition

$$
\lim _{r \nearrow \tau} \sigma(r) \neq \sigma(\tau)
$$

Assumption 7.1 may hold even when $\sigma$ does not have an atom at $\tau$.

Proposition 7.3. Suppose that 7.14 is valid; then $\mathcal{M}_{\natural} u(t)=\int_{-\tau}^{\tau} u(t+s) d \sigma(s)$ can be written

$$
\mathcal{M}_{\natural} u(t)=\eta u(\tau)+\int_{-\tau}^{\tau} u(t+s) d \delta(s) \quad(\eta \neq 0)
$$

where the variation $\mathrm{V}_{A}^{B}(\delta)$ (cf. Defn 3.2) satisfies $\lim _{\varepsilon \rightarrow 0+} \mathrm{V}_{\tau-\varepsilon}^{\tau}(\delta)=0$.

Remark 7.3. Recall that the functions $\sigma^{*}$, where $\sigma^{*}(r)=-\sigma(-r)$, generate $\mathcal{M}_{\natural}$ and $\mathcal{M}_{\theta}^{*}$ with the corresponding characteristic functions $\chi_{\mathcal{M}_{\sharp}}$ and $\chi_{\mathcal{M}_{\sharp}^{*}}$, and all the zeros of $\chi_{\mathcal{M}_{\sharp}^{*}}$ have non-zero imaginary part if and only if the zeros of the zeros of $\chi_{\mathcal{M}_{\natural}}$ have the same property. However, when $\sigma$ satisfies (7.14 there is (in general) no guarantee that the function $\sigma^{*}$ satisfies the corresponding requirement guaranteeing that $\mathcal{M}_{\sharp}^{*} \in \mathfrak{L}^{\sharp}$. In general,

$$
\mathcal{M}_{\natural} \in \mathfrak{L}^{\sharp} \not \mathcal{M}_{\sharp}^{*} \in \mathfrak{L}^{\sharp}
$$

We conclude that non-oscillatory in a weak sense and non-oscillatory are not equivalent properties. 


\section{Conclusions: Towards a Family of Canonical Equations for Oscillation}

As motivation, let us observe that advanced, retarded, and mixed type equations have very different properties, and without additional information on $d \sigma(s)$ the qualitative behaviour cannot be deduced if we work an equation of a different type. In the same way that the dynamics of the canonical ordinary differential equation

$$
u^{\prime}(t)=\lambda u(t)
$$

provides inadequate insight to the dynamics of the canonical retarded differential equation

$$
u^{\prime}(t)=\lambda u(t-1)
$$

so the retarded differential equation provides surprisingly little insight into the behaviour of the advanced or mixed-type equation.

Accordingly, the search for a single (or small class of) canonical problem that can be fully analysed and used to classify more general problems remains open. and will be the subject of further work.

Nevertheless, the authors take the view that the material reviewed and presented here provides simple methods to analyse oscillatory behaviour and also highlights the potential for misunderstanding and for drawing incorrect conclusions from an incomplete analysis. Further, the analysis of both oscillatory and stability behaviour for specific examples of equations of the form (*) in the Abstract is tractable using the approaches described here.

\section{Acknowledgements}

The authors are pleased to acknowledge the helpful comments of the anonymous referees which have led to an improved version of this paper.

\section{References}

[1] R. Bellman, K.L. Cooke, Differential Difference Equations, Academic Press, New York and London (1963).

[2] O. Diekmann, S. A. van Gils, S. M. Verduyn Lunel, H-O. Walther, Delay Equations Functional-, Complex-, and Nonlinear Analysis, Springer, New York (1995).

[3] J. M. Ferreira, S. Pinelas (2010), Oscillatory mixed differential systems, Funkcialaj Ekvacioj, 53, 1-20.

[4] J. M. Ferreira, N. J. Ford, M. A. Malique, S. Pinelas, Y. Yan (2011), Analytical and numerical treatment of oscillatory mixed differential equations with differentiable delays and advances, Journal of Computational and Applied Mathematics, 236, 5112-5130.

[5] N. J. Ford,, P. Lima, P., Lumb, P. M. and Teodoro, M. F., (2010), Analytical and numerical investigation of mixed-type functional differential equations, Journal of Computational and Applied Mathematics, 234, 2826-2837.

[6] L.D. Kudryavtsev (originator) Uniformly-convergent series, Encyclopedia of Mathematics, ISBN 1402006098. URL: http://www.encyclopediaofmath.org/index.php?title=Uniformlyconvergent_series\&oldid=30907 (2011) .

[7] J. K. Hale, , S. M. Verduyn Lunel,, Introduction to Functional Differential Equations, Springer-Verlag, New York (1993)

[8] T. Krisztyn (1994), Oscillation in linear functional differential systems, Differential Equations and Dynamical Systems, 2, 99-112.

[9] T. Krisztyn (1995), Exponential boundedness and oscillation for solutions of linear autonomous functional differential systems, Dynamic Systems and Applications, 4, 405-420. 
[10] T. K. Krisztin (2000), Nonoscillation for functional differential equations of mixed type, Journal of Math. Anal. Appl., 245, 326-345.

[11] S. Pinelas (2009), Oscillations and non-oscillations in mixed differential equations with monotonic delays and advances, Advances in Dynamical Systems and Applications, 4, 107-121.

[12] A. M. Pedro (2005), Oscillation and non-oscillation criteria for retarded functional differential equations, Proceedings of Equadiff -11, 1-10.

[13] E. C. Titchmarsh, Solutions of some functional equations, J. Lond. Math. Soc. (14) 1939, pp. $118-124$. 